\title{
Development of large readout area, high time resolution RPCs for LEPS2 at SPring-8
}

\author{
Natsuki Tomida ${ }^{1}$ \\ Department of physics, Kyoto University \\ Kitashirakawa-Oiwakecho, Sakyo-ku, Kyoto 606-8502, Japan \\ E-mail: natsukiescphys.kyoto-u.ac.jp
}

\section{Chia-Yu Hsieh}

Academia Sinica

128 Academia Road, Section 2, Nankang, Taipei 115, Taiwan

E-mail: cyhsiehlphys.sinica.edu.tw

\section{Masayuki Niiyama}

Department of physics, Kyoto University, Kyoto, Japan

\section{Hiroaki Ohnishi}

Nishina Center, RIKEN (The Institute of Physical and Chemical Research), Saitama, Japan

\section{Wen-Chen Chang}

Academia Sinica, Taipei, Taiwan

\section{Toshikazu Hashimoto}

Department of physics, Kyoto University, Kyoto, Japan

\section{Hirotomo Hamano}

Research Center for Nuclear Physics (RCNP), Osaka University, Osaka, Japan

\section{Keigo Mizutani}

Department of physics, Kyoto University, Kyoto, Japan

\begin{abstract}
We have developed prototype RPCs for the LEPS2 experiment at SPring-8 in Japan. At LEPS2, RPCs will be used as TOF detectors. The goal is to achieve a time resolution of 50 ps with a readout pad larger than $50 \mathrm{~cm}^{2} /$ channel. We made several prototypes and performed beam test at LEPS. A time resolution of 60 ps was obtained with $2.5 \mathrm{~cm} \times 40 \mathrm{~cm}$ readout pad.
\end{abstract}

XI workshop on Resistive Plate Chambers and Related Detectors (RPC2012)

INFN-Laboratori Nazionali di Frascati, Italy

February 5-10, 2012

\footnotetext{
$1 \quad$ Speaker
} 


\section{Introduction}

The construction of LEPS2 (Laser Electron Photon experiment at SPring-8), a new beamline for hadron photo-production experiments, has started at SPring-8 in Japan. The photon beam is produced by backward Compton scattering (BCS) of laser photons with $8 \mathrm{GeV}$ electrons circulating in SPring-8 storage ring. The energy range of photon beam is $1.5-3.0 \mathrm{GeV}$. Hyperons and vector mesons are produced by the photo-production. The LEPS experiment has reported various interesting data whereas the detection area was limited to forward angle [1,2]. The new LEPS 2 detectors will cover almost $4 \pi$ solid angle. Moreover, the beam intensity will be $10^{7} / \mathrm{s}$, which is one order of magnitude larger than LEPS.

In Fig. 1, a schematic drawing of LEPS2 detectors is shown. The solenoid magnet was moved from Brookhaven National Laboratory in the U.S., which was used for AGS E787/E949 experiment. RPCs will cover a barrel region of $5 \mathrm{~m}^{2}$ in the magnet. The flight length from the target is $1.0-1.7 \mathrm{~m}$. In order to separate kaons from pions up to $1.1 \mathrm{GeV} / \mathrm{c}$ with time-of-flight (TOF) measurement, $50 \mathrm{ps}$ TOF time resolution is required. Another important requirement is the number of channels. In order to reduce the number of channels to be less than 1000 , the readout area covered by one channel should be larger than $50 \mathrm{~cm}^{2}$. An efficiency better than 99\% is also required as RPCs will be used as trigger. Since the particles which arrive at the barrel part are mostly hadrons and the production rate of hadrons with $\gamma$-rays is quite low, a high rate capability is not required for our RPCs and the rate capability up to $1 \mathrm{~Hz} / \mathrm{cm}^{2}$ is sufficient.

\section{Prototype RPC}

We developed various types of RPCs with different gap width, number of stacks and readout pad size. The size of glass was $10 \mathrm{~cm} \times 50 \mathrm{~cm}$ and the thickness was $400 \mu \mathrm{m}$. A schematic drawing of the cross section of our typical RPC is shown in Fig. 2. Double-sided adhesive carbon tapes were used for electrodes. The surface resistivity of carbon tape was 500

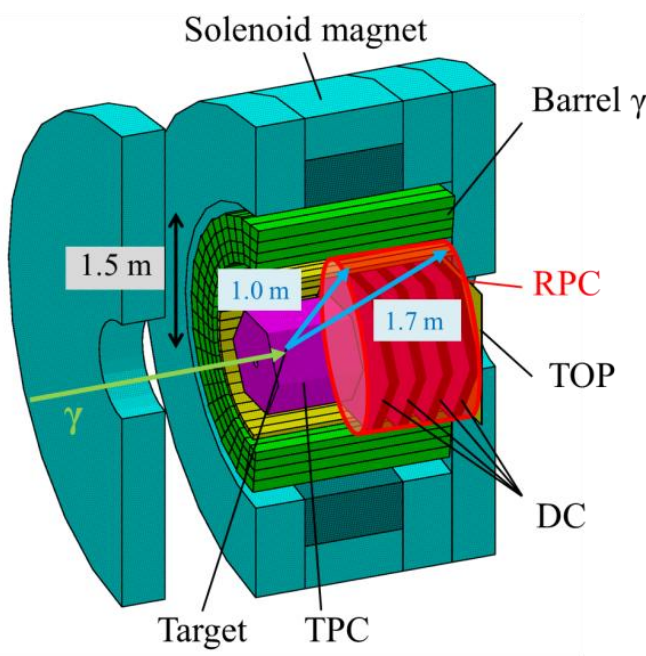

Fig. 1. Schematic drawing of LEPS2 detector. RPCs cover a barrel reagion. The radius of the barrel is $0.9 \mathrm{~m}$.

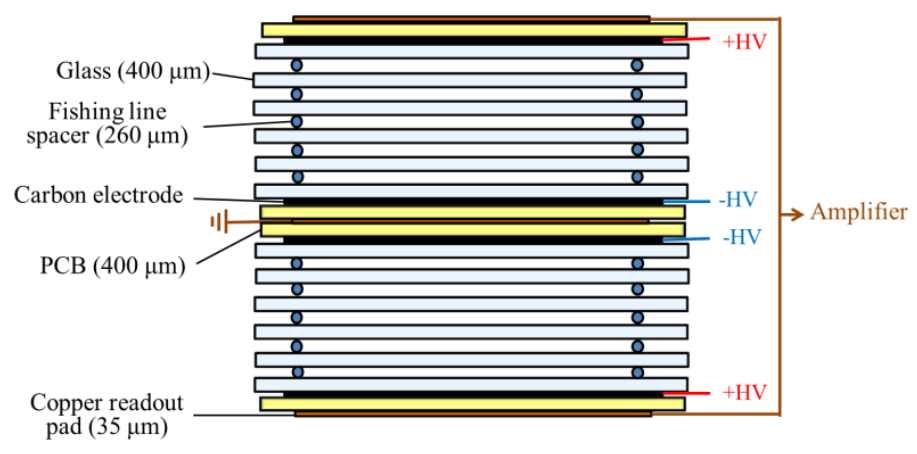

Fig. 2. Schematic drawing of a prototype RPC. The anode readout is connected to the amplifier and the cathode readout is connected to ground. 


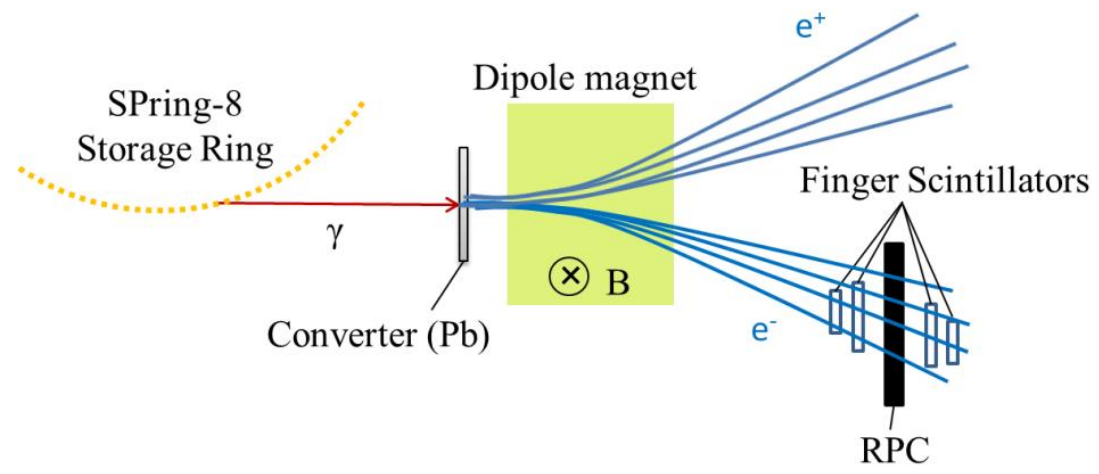

Fig. 3. Experimental set-up of beam test at LEPS. The $\gamma$-rays are converted to electron-positron pairs. Electrons are bent with a solenoid magnet and hit the RPC.

$\Omega / \square$. We used both $35 \mu \mathrm{m}$ thick copper tape and copper paint for readout pad. No difference of the resolution and the efficiency was observed using $1.5 \mathrm{~cm} \times 5.5 \mathrm{~cm}$ small pad. The gap width was defined by the diameter of fishing line used as a spacer and was $148 \mu \mathrm{m}$ and $260 \mu \mathrm{m}$. The number of stacks was one, two and four, and the number of gaps in one stack was defined so that the total voltage across a stack to be the same; 6 for $148 \mu \mathrm{m}$ and 5 for $260 \mu \mathrm{m}$ gap RPC. The anode pad was connected to the amplifier and the cathode pad was connected to ground. The gas mixture was $90 \% \mathrm{C}_{2} \mathrm{H}_{2} \mathrm{~F}_{4}, 5 \% \mathrm{SF}_{6}$ and $5 \%$ iso- $\mathrm{C}_{4} \mathrm{H}_{10}$.

\section{Experimental set up}

A beam test was performed at LEPS beamline. The experimental set-up is shown in Fig. 3. The BCS $\gamma$-rays were injected to a lead converter and electrons and positrons were produced with pair-creation. The electrons were bent with a dipole magnet and passed the RPC. The trigger area was defined with four finger scintillators as $1 \times 2 \mathrm{~cm}^{2}$. We kept trigger rate as $5-$ $20 \mathrm{~Hz} / \mathrm{cm}^{2}$ and this rate satisfied the requirement of LEPS2. We used a PMT amplifier, KN2104 manufactured by Kaizu Works, for signal amplification. The input impedance of the amplifier was $50 \Omega$. The data acquisition system was based on CAMAC. The TOF of electrons from the converter to the RPC was measured with a high resolution TDC module with 25 ps time bin, GNC-040 made by DNomes Design. The charge information was obtained with a Repic RPC022 ADC and slewing correction was applied for the time measurement. At LEPS and LEPS2, the RF signal is used as the start timing of TOF measurement. The RF signal is provided at the timing of the center of electron bunch in the SPring- 8 storage ring. Since the BCS photons flight at a certain (light) speed, the temporal position of electron which BCS is occurred is conserved. Therefore, the ambiguity of the timing of the pair creation at the converter is the time difference between the center of bunch and the electron which BCS was occurred. This time difference corresponds to the width of the electron bunch and it is 14-18 ps depending on the filling pattern of the electrons in the storage ring. 


\section{Test results}

Here we describe the time resolution and the efficiency of prototype RPCs obtained from beam test. We used a $1.5 \mathrm{~cm} \times 5.5 \mathrm{~cm}$ small pad to measure the gap width dependence and the number of stacks dependence of the time resolution. Then we moved to large readout pads. We show the pad size and the pad shape dependence of the time resolution. The time resolution shown here is over all TOF time resolution including the RF, front-end electronics and the RPC.

\subsection{Gap width dependence}

In Fig. 4, the time resolution and the efficiency of $1.5 \mathrm{~cm} \times 5.5 \mathrm{~cm}$ pad of $148-\mu \mathrm{m}$ and 260 $\mu \mathrm{m}$ gap width RPCs is shown. The RPCs were constructed as double stack configuration, and the total number of gaps was 12 and 10, respectively. The time resolution improved together with the increase of the efficiency but it becomes worse at higher voltage as the streamers appeared. Since not only the avalanche charge but also the streamer charge were measured with the ADC, the slewing correction did not work well at higher voltage. There was no big difference between $148-\mu \mathrm{m}$ and $260-\mu \mathrm{m}$ gap RPCs and both of them achieved 50 ps time resolution which satisfies the LEPS2 requirement though the pad was small.

\subsection{Number of stacks dependence}

We changed the number of stacks of $148-\mu \mathrm{m}$ and $260-\mu \mathrm{m}$ gap RPCs. In Fig. 5, the time resolution and the efficiency of $1.5 \mathrm{~cm} \times 5.5 \mathrm{~cm}$ pad are shown. By increasing number of stacks from one to two in 260- $\mu \mathrm{m}$ gap RPC, the resolution was improved about $10 \mathrm{ps}$ and the

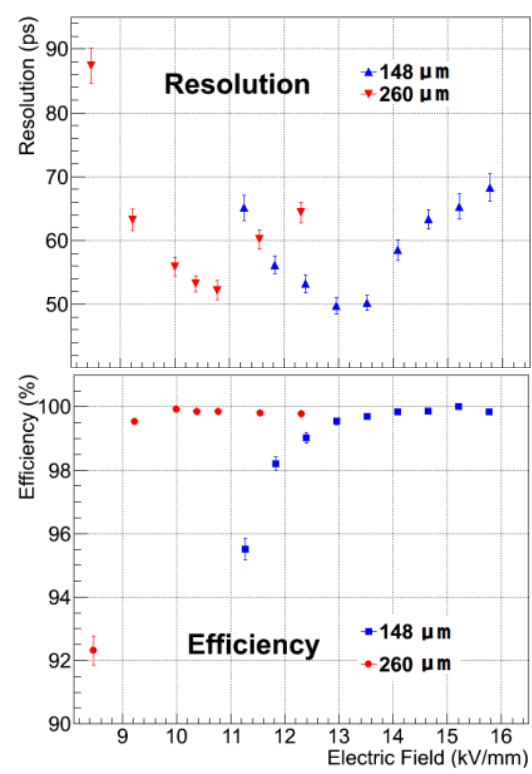

Fig. 4. The time resolution (up) and the efficiency (down) of the $148-\mu \mathrm{m}$ and the $260-\mu \mathrm{m}$ gap RPC as a function of applied voltage across the gap. There was no big difference between two RPCs on the performance.

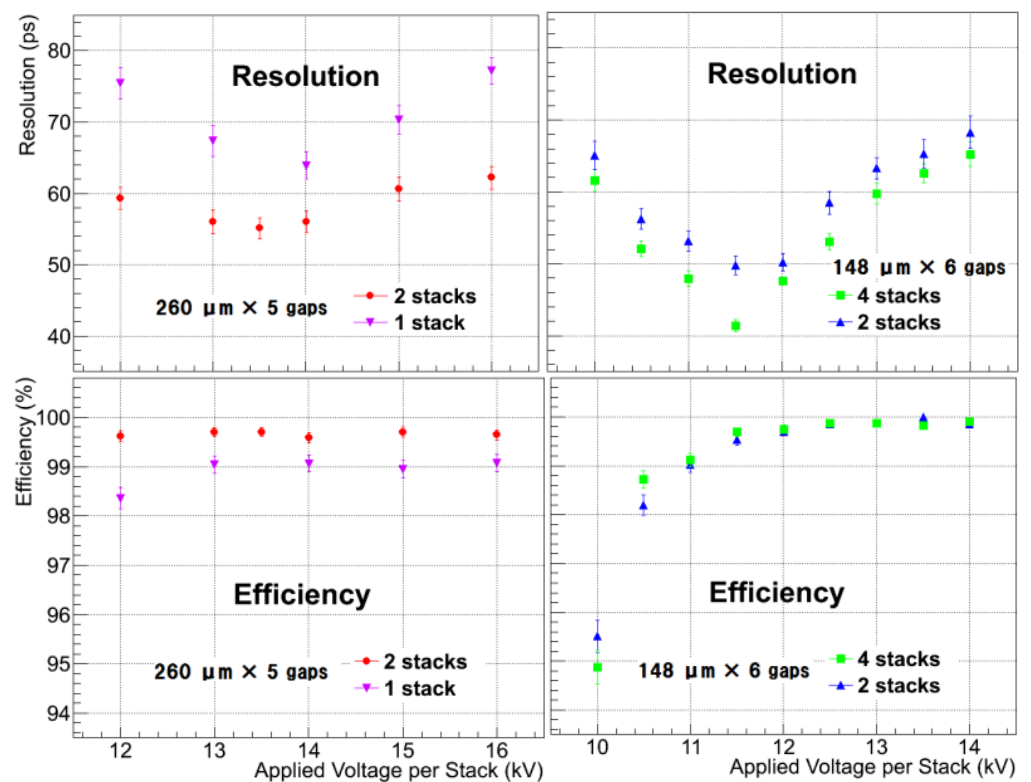

Fig. 5. The time resolution and the efficiency of the $260-\mu \mathrm{m}$ (left) and the $148-\mu \mathrm{m}$ gap RPC (right) in different stack configuration. The time resolution improved when the number of stacks is increased. 
efficiency reached better than $99 \%$. We also changed the number of stacks from two to four in $148-\mu \mathrm{m}$ gap RPCs. The resolution was improved about $5 \mathrm{ps}$ and achieved about $40 \mathrm{ps}$ at the best voltage value. There was no big difference between efficiencies of two RPCs because the twostack RPC already achieved an efficiency better than $99 \%$.

\subsection{Pad shape dependence}

\subsubsection{Rectangle pad}

We tested five different size readout pads on $148-\mu \mathrm{m}$ gap, double stack RPC. The smallest one was $1.5 \mathrm{~cm} \times 5.5 \mathrm{~cm}$, corresponding to 7000 channels if they are used at LEPS2 experiment and the largest was $10.0 \mathrm{~cm} \times 7.4 \mathrm{~cm}$, corresponding to 800 channels. Table 1 shows the resolution and the efficiency of each readout pad. Both the resolution and the efficiency become worse with increasing the pad size. We found it was difficult to achieve good resolutions with 'normal' rectangle-shaped large pads, then developed strip readout pads.

\subsubsection{Strip pad}

We developed three types of strip-shape readout pads on $148-\mu \mathrm{m}$ and $260-\mu \mathrm{m}$ gap with double stack configuration. The size of pad was $2.5 \mathrm{~cm} \times 20 \mathrm{~cm}, 1.5 \mathrm{~cm} \times 40 \mathrm{~cm}$ and $2.5 \mathrm{~cm} \times$ $40 \mathrm{~cm}$. We measured signals from both ends and averaged the timing. Table 2 shows the test results. During the measurement, the trigger position was set to be the center of the pads. The time resolutions were almost the same value for all pads. However, the efficiency of $148-\mu \mathrm{m}$ gap was low and only 260- $\mu \mathrm{m}$ gap RPC achieved close to $99 \%$ efficiency. The $2.5 \mathrm{~cm} \times 40 \mathrm{~cm}$ readout pad, corresponding to 1000 channels at LEPS2, achieved $60 \mathrm{ps}$ time resolution. These results are very close to the LEPS2 requirements.

The position dependence of the time resolution of $2.5 \mathrm{~cm} \times 40 \mathrm{~cm}$ readout pad is shown in Fig. 6. Although there was large position dependence on the resolution in each end, no big dependence was observed for the mean one. It is considered that the position dependence of the each readout resolution was resulted by the mismatch of the impedance between the strip readout and the amplifier. Therefore, it is necessary to develop an amplifier whose input impedance is matched with that of the strip readout.

\begin{tabular}{|c|c|c|c|}
\hline Readout Pad & Channel at LEPS2 & Resolution & Efficiency \\
\hline $1.5 \mathrm{~cm} \times 5.5 \mathrm{~cm}$ & 7000 & $50 \pm 1 \mathrm{ps}$ & $99.5 \pm 0.1 \%$ \\
\hline $2.5 \mathrm{~cm} \times 3.7 \mathrm{~cm}$ & 6500 & $57 \pm 1 \mathrm{ps}$ & $98.8 \pm 0.2 \%$ \\
\hline $2.5 \mathrm{~cm} \times 7.4 \mathrm{~cm}$ & 3000 & $65 \pm 3 \mathrm{ps}$ & $99.4 \pm 0.2 \%$ \\
\hline $5.0 \mathrm{~cm} \times 7.4 \mathrm{~cm}$ & 1500 & $85 \pm 3 \mathrm{ps}$ & $97.3 \pm 0.3 \%$ \\
\hline $10.0 \mathrm{~cm} \times 7.4 \mathrm{~cm}$ & 800 & $151 \pm 3 \mathrm{ps}$ & $90.0 \pm 0.5 \%$ \\
\hline
\end{tabular}

Table 1. The time resolution and the efficiency in different pad size. Both the time resolution and the efficiency become worse with increasing the pad size. 


\begin{tabular}{|c|c|c|c|c|}
\hline $\begin{array}{c}\text { Gap } \\
\text { width }\end{array}$ & Readout Pad & $\begin{array}{c}\text { Channel } \\
\text { at LEPS2 }\end{array}$ & Resolution & Efficiency \\
\hline \multirow{3}{*}{$148 \mu \mathrm{m}$} & $2.5 \mathrm{~cm} \times 20 \mathrm{~cm}$ & 2000 & $61 \pm 3 \mathrm{ps}$ & $92.8 \pm 0.4 \%$ \\
\cline { 2 - 5 } & $1.5 \mathrm{~cm} \times 40 \mathrm{~cm}$ & 1700 & $62 \pm 2 \mathrm{ps}$ & $96.4 \pm 0.3 \%$ \\
\cline { 2 - 5 } & $2.5 \mathrm{~cm} \times 40 \mathrm{~cm}$ & 1000 & $63 \pm 2 \mathrm{ps}$ & $90.4 \pm 0.5 \%$ \\
\hline \multirow{3}{*}{$260 \mu \mathrm{m}$} & $2.5 \mathrm{~cm} \times 20 \mathrm{~cm}$ & 2000 & $56 \pm 2 \mathrm{ps}$ & $99.2 \pm 0.2 \%$ \\
\cline { 2 - 5 } & $1.5 \mathrm{~cm} \times 40 \mathrm{~cm}$ & 1700 & $64 \pm 2 \mathrm{ps}$ & $98.4 \pm 0.3 \%$ \\
\cline { 2 - 5 } & $2.5 \mathrm{~cm} \times 40 \mathrm{~cm}$ & 1000 & $61 \pm 2 \mathrm{ps}$ & $98.6 \pm 0.2 \%$ \\
\hline
\end{tabular}

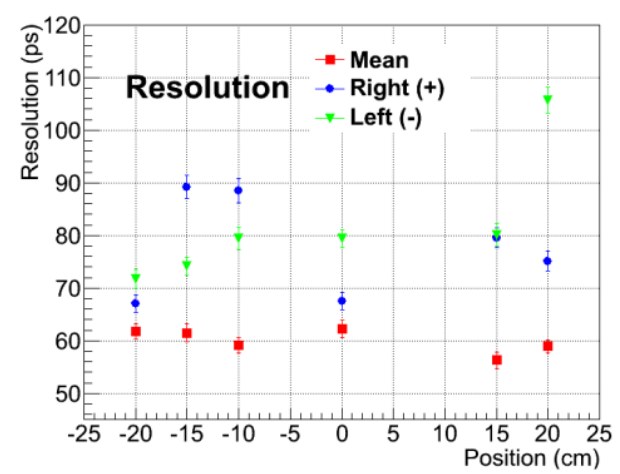

Fig. 6. The time resolution as a function of the position along $2.5 \mathrm{~cm} \times 40 \mathrm{~cm}$ strip readout pad. There was no position dependence with the mean time resolution while there was large position dependence with the each end resolution.

\section{Discussion}

The $2.5 \mathrm{~cm} \times 40 \mathrm{~cm}$ readout pad of $260-\mu \mathrm{m}$ gap RPC achieved $60 \mathrm{ps}$ TOF time resolution and $99 \%$ efficiency. This pad size corresponds to 1000 channels at LEPS2 and these results are very close to LEPS2 requirements. In order to improve the time resolution, we evaluated the contribution of each component in the TOF measurement to the time resolution. The effect of the propagation in the strip readout was obtained to be about 30 ps by subtracting the time resolution of $1.5 \mathrm{~cm} \times 5.5 \mathrm{~cm}$ pad $\left(\sigma_{\text {propagation }}^{2}=\sigma_{\text {strip }}^{2}-\sigma_{\text {small pad }}^{2}=60^{2}-52^{2}=30^{2}\right)$. The bunch width of electrons in the SPring- 8 storage ring, which corresponds to the resolution of the start timing, was 14 and 15 ps under the two filling pattern during the beam test. The time resolution of the TDC varied from 17 to $19 \mathrm{ps}$ according to the channel. The effect of the noise of the cable and the noise of the amplifier, were $10 \mathrm{ps}$ and $15 \mathrm{ps}$, respectively. The remaining contributions are the intrinsic time resolution of the RPC and the effect of the timing jitter of the amplifier and the discriminator. If we assume the resolution of the RPC as 15-25 ps [3], the effect of the amplifier and the discriminator is obtained to be 25-30 ps. Thus, the propagation in the strip readout and the timing jitter of the amplifier and the discriminator largely affect on overall TOF resolution. Therefore, it is important to develop a low-jitter amplifier which has impedance matched with the strip readout to reduce the two effects.

In the near future, we will develop a $110 \mathrm{~cm} \times 15 \mathrm{~cm}$ glass RPC, which is the same size as the one used at LEPS2 experiment. We will also develop low jitter and low noise amplifiers and TDCs. We plan to install our RPCs in 2013 and start physics data taking.

\section{References}

[1] T. Mibe, et al., Near-Threshold Diffractive $\phi-M e s o n$ Photoproduction from the Proton, Phys. Rev. Lett. 95 (2005) 182001

[2] T. Nakano, et al., Evidence for the $\Theta^{+}$in the $\gamma d \rightarrow K^{+} K$ pn reaction by detecting $K^{+} K$ pairs, Phys. Rev. C 79 (2009) 025210

[3] W. Riegler, et al., Detector physics and simulation of resistive plate chambers,

Nucl. Instr. and Meth. A $\mathbf{5 0 0}$ (2003) 144 